\title{
Specific Brainstem Neurons Switch Each Other into Pacemaker Mode to Drive Movement by Activating NMDA Receptors
}

\author{
Wen-Chang Li, ${ }^{1,2}$ Alan Roberts, ${ }^{2}$ and Stephen R. Soffe ${ }^{2}$ \\ ${ }^{1}$ School of Biology, University of St. Andrews, St. Andrews, Fife KY16 9TS, United Kingdom, and ${ }^{2}$ School of Biological Sciences, University of Bristol, Bristol \\ BS8 1UG, United Kingdom
}

\begin{abstract}
Rhythmic activity is central to brain function. In the vertebrate CNS, the neuronal circuits for breathing and locomotion involve inhibition and also neurons acting as pacemakers, but identifying the neurons responsible has proven difficult. By studying simple hatchling Xenopus laevis tadpoles, we have already identified a population of electrically coupled hindbrain neurons (dINs) that drive swimming. During rhythm generation, dINs release glutamate to excite each other and activate NMDA receptors (NMDARs). The resulting depolarization enables a network mechanism for swimming rhythm generation that depends on reciprocal inhibition between antagonistic right and left sides. Surprisingly, a surgically isolated hemi-CNS without inhibition can still generate swimming-like rhythms. We have now discovered that activation of NMDARs transforms dINs, which normally fire singly to current injection, into pacemakers firing within the normal swimming frequency range $(10-25 \mathrm{~Hz})$. When dIN firing is blocked pharmacologically, this NMDAR activation produces $10 \mathrm{~Hz}$ membrane potential oscillations that persist when electrical coupling is blocked but not when the voltage-dependent gating of NMDARs by $\mathrm{Mg}^{2+}$ is removed. The NMDA-induced oscillations and pacemaker firing at swimming frequency are unique to the dIN population and do not occur in other spinal neurons. We conclude that NMDAR-mediated self-resetting switches critical neurons that drive swimming into pacemaker mode only during locomotion where it provides an additional, parallel mechanism for rhythm generation. This allows rhythm generation in a half-CNS and raises the possibility that such concealed pacemaker properties may be present underlying rhythm generation in other vertebrate brain networks.
\end{abstract}

\section{Introduction}

Rhythmic activity is widespread in the vertebrate CNS controlling functions like locomotion, respiration, sleep, and memory consolidation (for review, see Buzsáki and Draguhn, 2004; Ramirez et al., 2004). Rhythm generating networks rely on both synaptic connections between network neurons and the cellular properties of the neurons (Getting, 1989; Harris-Warrick, 2002; Arshavsky, 2003; Ramirez et al., 2004; Guertin, 2009; Brocard et al., 2010). Vertebrate locomotion requires rhythmic patterned activation of trunk and limb muscles. Although spinal networks can organize such locomotor rhythms, it is generally assumed that descending excitation from hindbrain reticulospinal neurons is necessary to activate NMDA receptors (NMDARs) and turn on the spinal rhythms (Arshavsky et al., 1984; Atsuta et al., 1990; Noga et al., 1991). To investigate whether pacemaker properties are involved in rhythm generation, tetrodotoxin (TTX) has been used in many preparations to block impulses. NMDA induces slow (typically $\sim 1 \mathrm{~Hz}$ ) membrane potential oscillations in

\footnotetext{
Received July 8, 2010; revised Sept. 10, 2010; accepted Oct. 5, 2010.

This work was supported first by a Wellcome Trust grant (A.R., S.R.S.) and then by a Royal Society University Research Fellowship (W.-C.L.). We thank Dr. Matt Jones and Dr. Marc Holderied for commenting on previous versions of this paper. W.-C.L. performed most experiments and data analyses. W.-C.L., A.R., and S.R.S. contributed to the conception and design of experiments, interpretation of data, and manuscript writing.

Correspondence should be addressed to Wen-Chang Li at the above address. E-mail: w121@st-andrews.ac.uk. DOI:10.1523/JNEUROSCI.3695-10.2010

Copyright $\odot 2010$ the authors $\quad 0270-6474 / 10 / 3016609-12 \$ 15.00 / 0$
}

motoneurons and some interneurons, which may contribute to their rhythmic firing during locomotion (Grillner and Wallén, 1985; Wallén and Grillner, 1985, 1987; Hochman et al., 1994a,b; Sillar and Simmers, 1994; Rioult-Pedotti, 1997; Guertin and Hounsgaard, 1998; Reith and Sillar, 1998; Prime et al., 1999; Tresch and Kiehn, 2000; Wilson et al., 2005).

In hatchling Xenopus tadpoles, we have recently established that one type of reticulospinal neuron (dINs) provides the excitatory synaptic drive to spinal neurons during swimming locomotion. The reliable generation of the swimming rhythm depends, first, on dINs exciting each other by releasing glutamate to activate AMPA receptors (AMPARs) and NMDARs; second, on rebound firing in the dINs after reciprocal inhibition (Li et al., 2006; Soffe et al., 2009); and third, on the dINs being electrically coupled to each other ( $\mathrm{Li}$ et al., 2009). However, a swimming-like rhythm of motor activity at $15-25 \mathrm{~Hz}$ can also be produced by a single, surgically isolated side of the CNS (hemi-CNS) even when inhibition is blocked by antagonists (Soffe, 1989). Single-sided swimming rhythms have also been found in the lamprey (Cangiano and Grillner, 2003, 2005). Similarly, the pharmacological block of glycinergic transmission does not stop the generation of rhythmic activity in either intact cord or hemicord preparations in the mouse (Droge and Tao, 1993; Cowley and Schmidt, 1995; Ozaki et al., 1996; Kremer and Lev-Tov, 1997) and adult frog (Rioult-Pedotti, 1997). These results suggest that other, possibly cellular pacemaker mecha- 
nisms are present that do not depend on inhibition. Our aim is to reveal pacemaker properties in the tadpole swimming circuit that could contribute to normal network rhythm generation and also allow a single side of the CNS to generate rhythm.

We found that the application of NMDA can indeed transform the reticulospinal dIN neurons that drive swimming into pacemakers. Since dINs synapse with each other and release glutamate to activate NMDARs, this suggests a novel role for NMDAR activation to self-reset the firing properties within a neuronal population while it is in an active state.

\section{Materials and Methods}

Preparation. Human chorionic gonadotropin injections were performed regularly in our $\mathrm{Xe}$ nopus colony to induce mating. Embryos were collected and incubated at different temperatures to alter their developmental rates. All experiment procedures have been approved by local Animal Welfare Ethics committee and comply with United Kingdom Home Office regulations. Xenopus tadpoles [stage 37/38 (Nieuwkoop and Faber, 1956)] were briefly anesthetized using $0.1 \%$ MS222 (3-aminobenzoic acid ester) (Sigma-Aldrich), and then immobilized using $10 \mu \mathrm{M} \quad \alpha$-bungarotoxin (Tocris Bioscience) and mounted onto a small Sylgard stage using fine tungsten pins for dissection as described previously (Li et al., 2002). The saline had the following concentrations (in mм): $115 \mathrm{NaCl}, 3 \mathrm{KCl}, 2 \mathrm{CaCl}_{2}, 2.4 \mathrm{NaHCO}_{3}, 1$ $\mathrm{MgCl}_{2}, 10$ HEPES, adjusted to $\mathrm{pH} 7.4$ with $\mathrm{NaOH}$. Once the CNS was exposed (see Fig. $1 A-C$ ), the hemi-CNS preparation was made by cutting through the cord caudally at the eighth to ninth muscle segment and the hindbrain at the level of the ear vesicle (fifth to sixth rhombomere segments). The left side of the hindbrain and spinal cord was then removed between these cuts (see Fig. 1D). The nervous system was then opened and tissue removed to expose neuronal somata to allow access of whole-cell recording electrodes.

Recordings. After dissection, whole-cell current-clamp recordings were made from exposed neuronal somata using patch pipettes filled with $0.1 \%$ Neurobiotin (Vector Laboratories) in the intracellular solution (concentrations in mM: $100 \mathrm{~K}$-gluconate, $2 \mathrm{MgCl}_{2}, 10 \mathrm{EGTA}, 10$ HEPES, $3 \mathrm{Na}_{2} \mathrm{ATP}, 0.5 \mathrm{NaGTP}$, adjusted to $\mathrm{pH} 7.3$ with $\mathrm{KOH}$ ). Pipettes had DC resistances ranging from 10 to $20 \mathrm{M} \Omega$. Whole-cell recordings were mostly made using Axon Multiclamp 700B, digitized with Digidata 1440 A, sampled with pClamp 10 software (Molecular Devices). Some whole-cell recordings were made with Axon-2B amplifier, CED 1401 plus digitizer, and Signal software (Cambridge Electronic Design). Tip potentials were corrected before making recordings. Microperfusion of NMDA or L-glutamate (Sigma-Aldrich) was done by using a glass pipette with an opening of $10 \mu \mathrm{m}$ at the tip positioned as close as $30 \mu \mathrm{m}$ to the recorded soma. To test the effects of NMDA on dIN properties (firing, oscillations), the level of NMDAR activation was adjusted by changing either the perfusion pressure or the distance of the microperfusion pipette from the recorded neuron somata. In this case, the actual concentration of NMDA reaching its sites of action can vary from trial to trial and will be lower than the concentration in the pipette because of dilu-
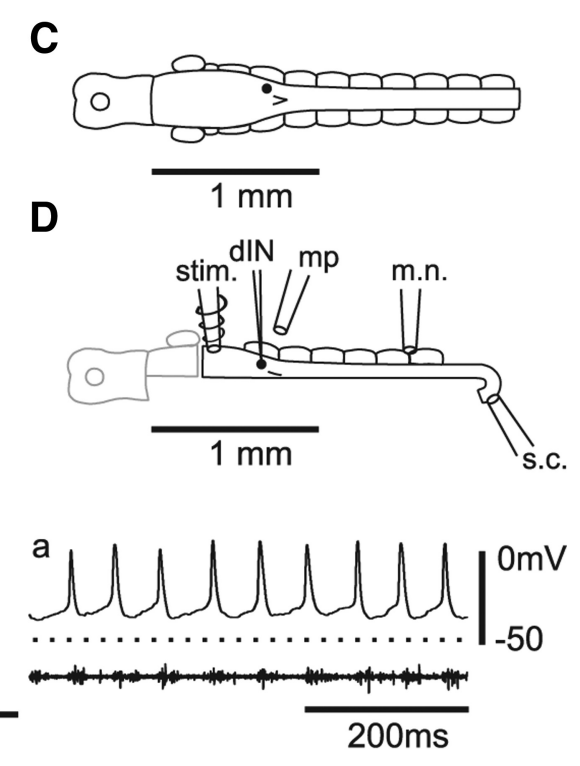

2s
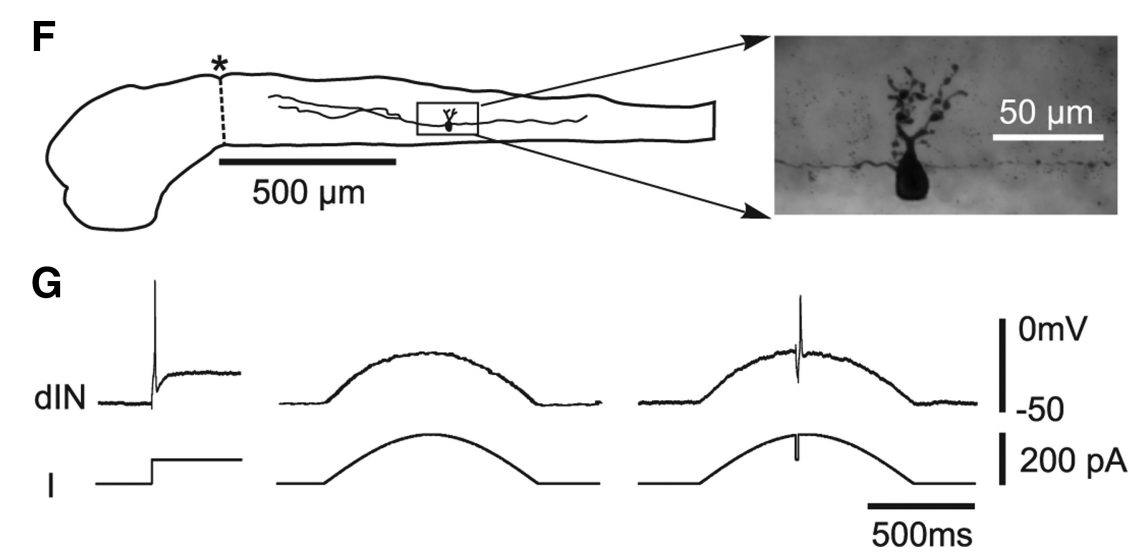

Figure 1. Diagrams of the tadpole, experimental setup, activity during swimming, and dIN anatomy. $A$, Tadpole at stage $37 / 38$. $B$, Tadpole in a side view to show CNS (shaded). The asterisk (*) marks caudal end of hindbrain. $C$, Top view of tadpole CNS and swimming myotomes. D, Hemi-CNS preparation. Electrodes and pipettes: stim., stimulation; $\mathrm{dIN}$, whole cell; mp, microperfusion ., motor nerve; S.C., spinal cord. $\boldsymbol{E}$, dIN activity during light dimming evoked swimming-like rhythm recorded directly from the reliably at the onset of square current injections (left) fails to fire any spike to slowly rising depolarization (middle) but can fire after a reduction of slow sinusoidal current injection (I) for $25 \mathrm{~ms}$.

tion with saline. In a few experiments, NMDA or L-glutamate (both at $100 \mathrm{~mm}$ prepared with equimolar sodium hydroxide) was applied by microiontophoresis. The microiontophoresis electrodes had DC resistances of $150-200 \mathrm{M} \Omega$ when filled with $3 \mathrm{~m}$ potassium acetate and were placed within $50 \mu \mathrm{m}$ upstream to the recording site. Pulses of negative current $<2.5 \mathrm{nA}$ were configured using pClamp10 and injected through the second channel of the MultiClamp 700B. Simply positioning the microiontophoresis electrode close to the recorded neuron did not cause a membrane potential change so holding currents were not used. In immobilized tadpoles, fictive swimming was evoked by $1 \mathrm{~ms}$ current pulse stimulation to the skin, dimming the microscope light, or, in some hemi-CNS preparations, stimulating the hindbrain directly. Motor nerve recordings were made by placing a suction electrode on the segmented swimming muscle clefts to monitor spinal cord outputs. In preparations in which TTX (Sigma-Aldrich) or $\mathrm{Cd}^{2+}$ was used to block action potential triggered synaptic transmission, the half-hindbrain was left intact so light dimming could be used to evoke swimming-like rhythms so active dINs could be found using extracellular recording. In many hemi-CNS preparations, NMDA was microperfused onto the caudal hindbrain/rostral spinal cord region to initiate rhythmic activity. Such activity was 


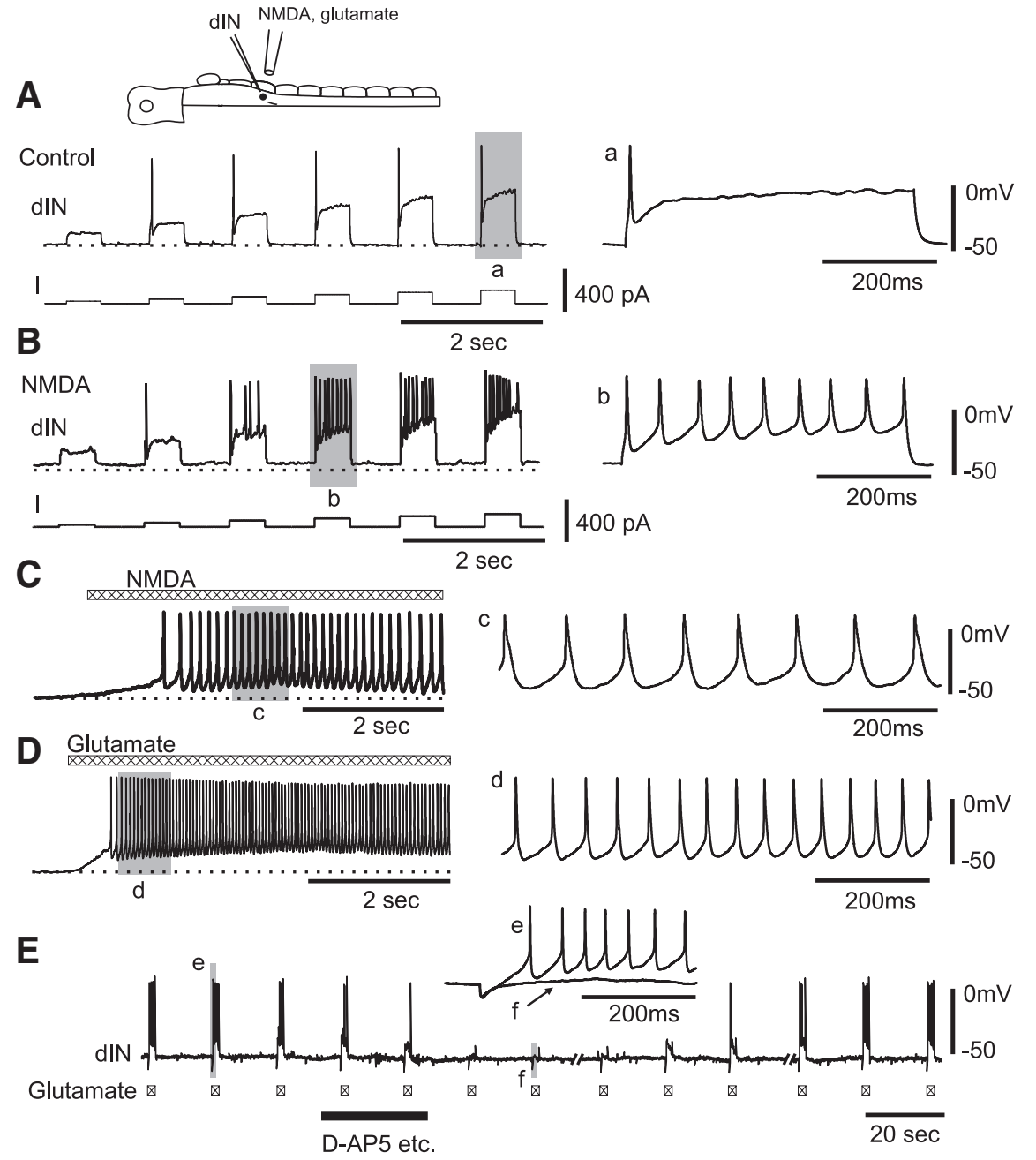

Figure 2. dINs fire repetitively to NMDA and glutamate application. $A$, At rest, a dIN fires single action potentials to current injections. $\boldsymbol{B}$, dIN in $\boldsymbol{A}$ fires repetitively when $100 \mu \mathrm{m} \mathrm{NMDA}$ applied by microperfusion depolarized the membrane potential by $\sim 5$ $\mathrm{mV}$ from rest (dotted line). C, Repetitive dIN firing response to microperfusion of NMDA alone. $\boldsymbol{D}$, Another dIN fired repetitively to microperfusion of $1 \mathrm{~mm}$ glutamate. In Cand D, saline contained $1 \mu \mathrm{m}$ strychnine, $20 \mu \mathrm{mSR} 95531,5 \mu \mathrm{m} \mathrm{NBQX}, 2 \mu \mathrm{m} \mathrm{DH} \beta \mathrm{e}$, and 150 $\mu \mathrm{M} \mathrm{Cd}^{2+}$.E, The responses of a dIN to L-glutamate applied via a microiontophoresis electrode $(-0.8 \mathrm{nA}, \sim 20 \mu \mathrm{m}$ away from dIN $)$ before, during, and after antagonist mixture (D-AP5, etc.: $50 \mu \mathrm{m} \mathrm{D}-\mathrm{AP5} 5,1 \mu \mathrm{m}$ strychnine, $20 \mu \mathrm{mSR} 95531,5 \mu \mathrm{m} \mathrm{NBQX,2} \mu \mathrm{m}$ DH $\beta \mathrm{e}$ ) dropped into a small well upstream to the recording chamber. Saline contained $150 \mu \mathrm{M} \mathrm{Cd}^{2+}$. Shaded area records $\boldsymbol{a}-\boldsymbol{e}$ and $\boldsymbol{f}$ are expanded on the right.

monitored either by making motor nerve recordings or by recording directly from the caudal hemicord using a suction electrode (see Fig. 1). When 18- $\beta$-glycyrrhetinic acid (18- $\beta$-GA) (Sigma-Aldrich) was used to block the electrical coupling, $100 \mu \mathrm{M}$ NMDA was bath-applied to induce oscillation. In this case, pairs of dINs were recorded in perforated wholecell recording mode using amphotericin B (100 $\mu \mathrm{g} / \mathrm{ml}$; Merck4Biosciences) to facilitate stable recordings in both neurons. Electrical coupling coefficient was defined as the percentage of voltage response in the uninjected neuron relative to the response in the injected neuron. Neuronal anatomy revealed by Neurobiotin filling was used for final neuronal identification. Transmitter antagonists (full name; receptor type) used included the following: strychnine (GlyR); 6-imino-3-\{4-methoxyphenyl $\}-1\{6 H\}$-pyridazinebutanoic acid hydrobromide (SR95531; also called gabazine) $\left(\mathrm{GABA}_{\mathrm{A}} \mathrm{Rs}\right)$; 2,3dihydroxy-6-nitro-7-sulfamoylbenzo-[f]quinoxaline (NBQX) (AMPAR); D-(-)-2-amino-5-phosphonopentanoic acid (D-AP5) (NMDAR) (Tocris Bioscience); and dihydro- $\beta$-erythroidine ( $\mathrm{DH} \beta \mathrm{e}$ ) (nAChRs) (Research Biochemicals International).

Data analyses. These were performed with Dataview (version 6.1, courtesy of Dr. W. J. Heitler, University of St. Andrews, St. Andrews, Fife, UK) and SPSS. To classify neuronal responses to NMDA application in the presence of TTX, autocorrelation was applied to the recordings to extract the oscillation frequency [the inverse of the time $(T)$ to the first autocorrelation peak] and measurements of the first autocorrelation peak and trough. Cluster analyses using oscillation amplitude, frequency, and the difference between the first autocorrelation trough and peak measurements were used to classify neurons using SPSS. Power spectrum analyses were performed using $\mathrm{dIN}$ recordings to compare the frequency composition of the oscillations. Phase plots were used to evaluate the synchronicity of oscillations in the simultaneously recorded dINs. To evaluate rhythmicity of either motor nerve or spinal cord bursts, the records were rectified first. Then a threshold amplitude was set to trigger events and histograms of intervals between these events produced using Dataview. Coefficients of rhythmicity were calculated as follows: $\mathrm{Cr}=$ $(\mathrm{a} 1-\mathrm{a} 2) /(\mathrm{a} 1+\mathrm{a} 2)$, where $\mathrm{a} 1$ and $\mathrm{a} 2$ are the sizes of the second peak and first trough of the histogram, respectively (Thörn Pérez et al., 2009) (see Fig. 9B). Similar measurements were made of events triggered by action potentials in whole-cell recordings from individual neurons. Means were compared using $t$ tests unless stated otherwise.

\section{Results}

Neurons in the caudal hindbrain were identified as reticulospinal dINs during experiments by their unusually long duration action potentials and single spike per cycle firing during swimming-like activity (Fig. $1 E$ ) (Li et al., 2006). They were filled with Neurobiotin and the identification confirmed if they possessed a descending ipsilateral axon (Fig. 1F). The dINs recorded in this paper shared the same properties, physiology, activity during swimming, and anatomy as dINs and hdINs (hindbrain dINs) already extensively documented (Li et al., 2004, 2006, 2009; Soffe et al., 2009). Significantly, they typically fire just a single action potential at the onset of a step depolarizing current injection, even well above threshold (Figs. 1G, 2A). Only interrupting the depolarization with negative current or synaptic inhibition can make them fire again on rebound (Soffe et al., 2009). However, these dINs do not fire to slowly rising depolarization. When tested in eight dINs, slowly rising ramp or $0.5 \mathrm{~Hz}$ sinusoidal current injection did not produce spikes even when neurons were depolarized to membrane potentials $(-10.5 \pm 6.3 \mathrm{mV})$ well above their firing thresholds measured in response to square current pulses $(-30 \pm 4.5 \mathrm{mV}$ ) (Fig. $1 G$ ). Firing could still be induced in slowly depolarized dINs on rebound from a negative shift in potential.

\section{NMDA induced repetitive firing to current in reticulospinal dINs}

During swimming, reticulospinal dINs in the caudal hindbrain receive glutamatergic excitation from other dINs, which activates both AMPAR and NMDAR and summates to provide a sustained background excitation ( $\mathrm{Li}$ et al., 2006). Here, we tested whether 
NMDA application could additionally induce any pacemakerlike properties in dINs.

We examined the effects of NMDAR activation by using a pipette with $\sim 10 \mu \mathrm{m}$ tip opening to microperfuse $100 \mu \mathrm{M}$ NMDA onto dINs (Fig. 1D) with $1 \mu \mathrm{M}$ strychnine and $20 \mu \mathrm{M}$ SR95531 in the saline. By adjusting the microperfusion pressure and pipette distance from the recorded cell body, we were able to microperfuse sufficient NMDA to slightly depolarize the membrane potential (typically by $5 \sim 10 \mathrm{mV}$ ). This small depolarization makes it clear that at the dIN resting potential, NMDARs are not fully blocked by $\mathrm{Mg}^{2+}$ (cf. Mayer et al., 1984; Soffe and Roberts, 1989). Interestingly, when this was done, 15 of 16 single firing dINs started to fire repetitively in response to current injection (Fig. $2 \mathrm{~B}$ ). In seven dINs, a series of currents were injected to investigate the firing frequency range. This was very narrow and lay mainly within the normal tadpole swimming frequency range (7-27.4 Hz; mean, $17.8 \pm 1.7 \mathrm{~Hz}$; one dIN with a wider range of 19-44 Hz) (Fig. 3B). Bigger NMDA depolarization even without current injection always led to repetitive $\operatorname{dIN}$ firing $(n=$ 15 dINs) often followed by rhythmic network activity. To avoid such activity and test whether the NMDA was acting directly on dINs to induce repetitive firing, we used $150 \mu \mathrm{M} \mathrm{Cd}{ }^{2+}$ to globally block synaptic transmission and $1 \mu \mathrm{M}$ strychnine, 20 $\mu \mathrm{M}$ SR95531, $2 \mu \mathrm{M}$ DH $\beta \mathrm{e}, 5 \mu \mathrm{M}$ NBQX to block specific ionotropic receptors. In this case, NMDA induced dIN firing with frequency ranging from 5 to $30 \mathrm{~Hz}$ (mean of averages: $16.9 \pm$ $2.6 \mathrm{~Hz}, n=7$ dINs, 1192 spikes analyzed) (Figs. 2C, 3A). Although dINs were previously shown to corelease ACh along with glutamate (Li et al., 2004), repetitive firing in the presence of the antagonist $\mathrm{DH} \beta \mathrm{e}$ demonstrates that nicotinic receptor activation is not necessary for the transformation of dIN firing properties.

Since dINs release glutamate, we microperfused $1 \mathrm{~mm}$ L-glutamate while using $1 \mu \mathrm{M}$ strychnine, $20 \mu \mathrm{M}$ SR95531, $2 \mu \mathrm{M}$ $\mathrm{DH} \beta \mathrm{e}, 5 \mu \mathrm{M} \mathrm{NBQX}$, and $150-200 \mu \mathrm{M} \mathrm{Cd}^{2+}$ to block other ionotropic receptors. When glutamate-induced depolarizations were small, dINs started to fire repetitively to injected current. Larger glutamate-induced depolarizations made dINs fire repetitively without additional current (Fig. 2D). The dIN firing frequencies to glutamate applications (mean of average: $16.4 \pm 1.9 \mathrm{~Hz}, n=5$ dINs, 680 spikes analyzed) are similar to those seen with NMDA applications (Fig. $3 A)(p=0.74)$. L-Glutamate $(n=5)$ or NMDA $(n=1,-0.05$ to $-2.5 \mathrm{nA})$ applied by microiontophoresis also revealed that repetitive dIN firing can be evoked within $<200 \mathrm{~ms}$ (from microiontophoresis current onset to first spike peak: $179 \pm 95 \mathrm{~ms}$; shortest, $81 \mathrm{~ms}$ ) (Fig. $2 \mathrm{E}$ ), equivalent to a few swimming cycles. When $50 \mu \mathrm{M}$ D-AP5 was included in the antagonist mixture, glutamate-induced membrane depolarization was mostly blocked and glutamate could not make dINs fire $(n=7)$ (Fig. 2E), further confirming that the activation of NMDARs rather than other ionotropic or metabotropic glutamate receptors is necessary to induce repetitive dIN firing.

The NMDA-induced firing of dINs has two significant features for their possible role as conditional pacemaker neurons in the swim rhythm generator: first, the firing rates of dINs in NMDA were restricted and were increased little by increasing current injection, and second, the firing rates lay mostly within the natural $10-25 \mathrm{~Hz}$ range of swimming frequencies seen in the tadpole (Fig. 3B) (Kahn et al., 1982). In contrast, other spinal neurons that are active during swimming $(n=9)$ fired repetitively to positive current injections at frequencies up to $200 \mathrm{~Hz}$, which are considerably higher than those of swimming (Fig. 3C) (Sautois et al., 2007). These neurons also fired repetitively at high

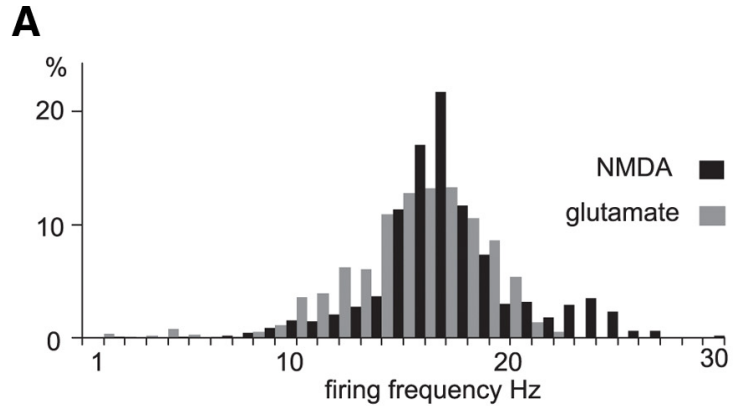

B
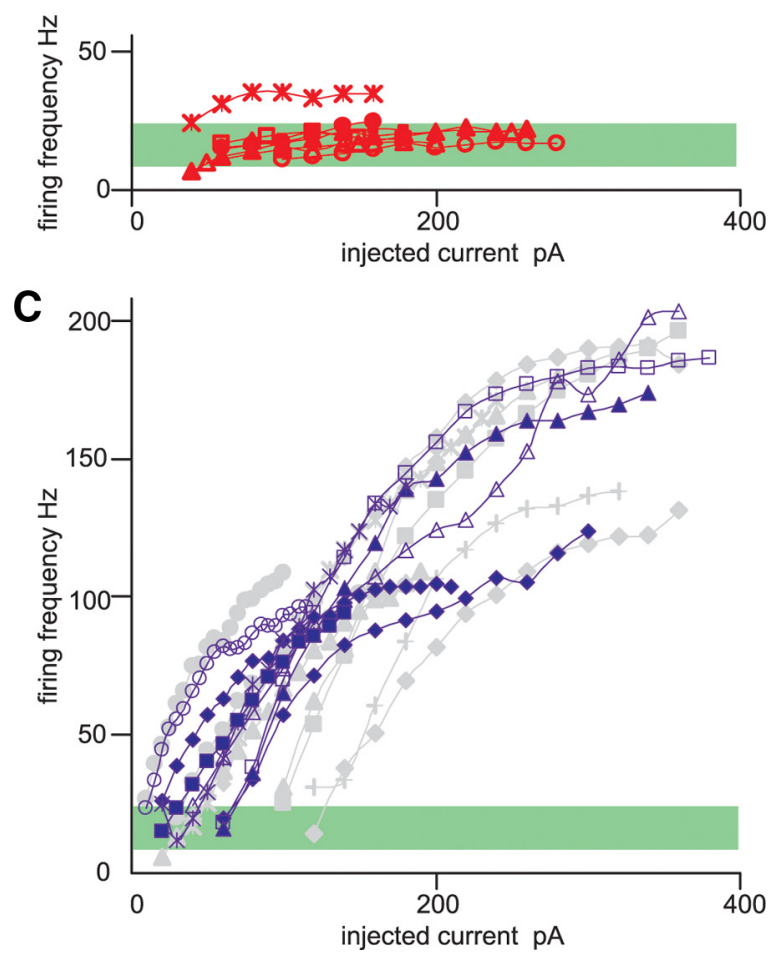

Figure 3. The frequency range of NMDA-induced firing. $A$, Comparison of dIN firing frequency distributions evoked by NMDA and glutamate applications in the presence of $1 \mu \mathrm{M}$ strychnine, $20 \mu \mathrm{m}$ SR95531, $2 \mu \mathrm{m}$ DH $\beta \mathrm{e}, 5 \mu \mathrm{m}$ NBQX, and 150-200 $\mu \mathrm{m} \mathrm{Cd}^{2+}$ (NMDA, 1192 spikes from $7 \mathrm{dINs}$; glutamate, 680 spikes from $5 \mathrm{dINs}$ ). Counts of spikes per frequency bin were normalized to percentages for each neuron and averaged across the sample. $\boldsymbol{B}, \boldsymbol{C}$, Effects of current level on neuronal firing in the presence of NMDA in dINs (red symbols and lines in $\boldsymbol{B}$ ) and non-dINs (blue in C). The gray symbols in ( show non-dIN firing in control saline. The green area shows normal swim frequency range $(10-25 \mathrm{~Hz})$.

frequencies to current injection when their membrane potential was depolarized by microperfused NMDA (Fig. 3C) (5.2-14.4 $\mathrm{mV}$ from rest). So, unlike dINs, firing characteristics of other spinal neurons are not noticeably changed by NMDA.

\section{NMDA induced oscillations in reticulospinal dINs}

Our next step was to examine what happens in the presence of NMDA to make single-firing dINs into potential pacemakers that fire repetitively within the swimming frequency range. Previous work on embryonic Xenopus spinal neurons had revealed NMDA induced fast membrane potential oscillations when action potentials were blocked by TTX (Prime et al., 1999). We therefore used $0.4 \mu \mathrm{M}$ TTX to exclude spikes and therefore spike-evoked chemical synaptic transmission and then applied NMDA to see whether oscillations were induced. Both microperfused and bath-applied NMDA $(20-100 \mu \mathrm{M})$ induced clear membrane potential oscillations in 75 of 77 dINs recorded (Fig. $4 A$ ). When 


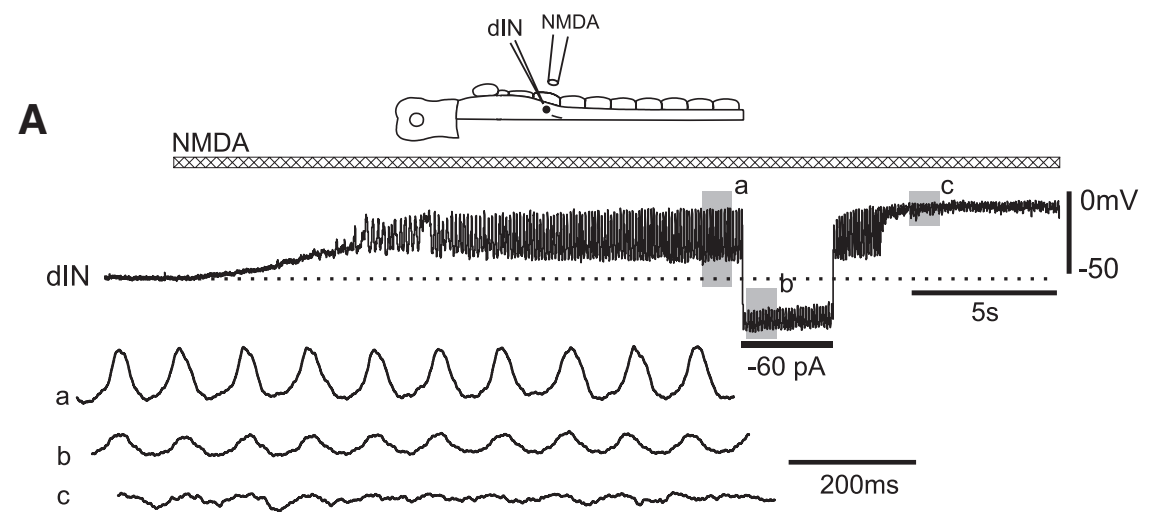

B
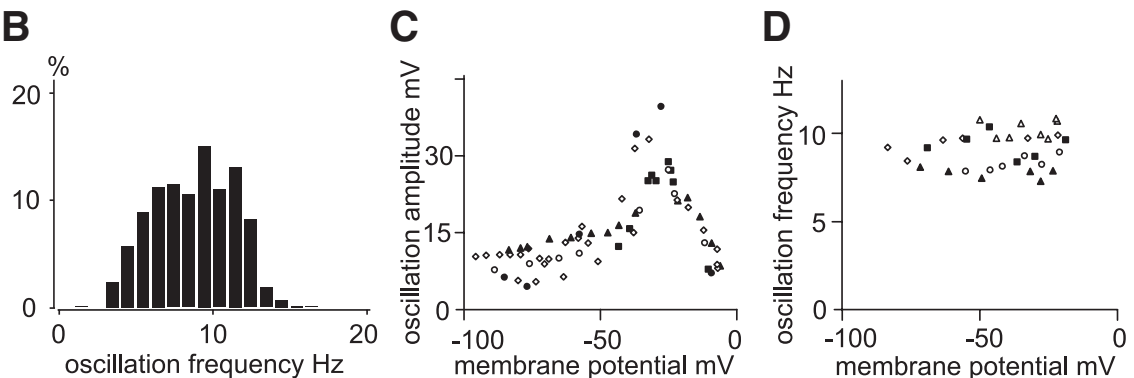

Figure 4. NMDA-induced dIN oscillations in TTX. $\boldsymbol{A}$, Depolarization and membrane potential oscillations in a dIN during NMDA application (bar) in TTX. Shaded areas $\boldsymbol{a}$ - $\mathbf{c}$ are expanded below to show oscillations. $\boldsymbol{B}$, Frequency distribution of TTX-resistant oscillations in dINs evoked by microperfused NMDA (1589 cycles from $10 \mathrm{dINs}$ ). Counts of cycles per frequency bin were normalized to percentages for each neuron and averaged across the sample. $\boldsymbol{C}$, The relationship between the amplitude of NMDA-induced TTX-resistant oscillations and membrane potential changed by current injections. $\boldsymbol{D}$, The relationship between oscillation frequency and half-oscillation height membrane potential levels when NMDA was bath-applied. The different symbols indicate different dINs.

A

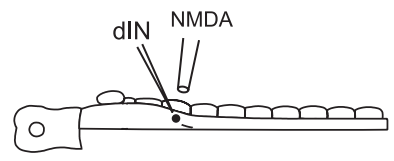

NMDA, $\mathrm{Mg}^{2+}$

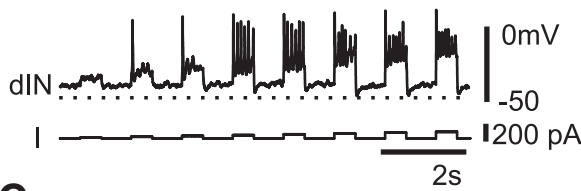

C NMDA, $0 \mathrm{Mg}^{2+}$

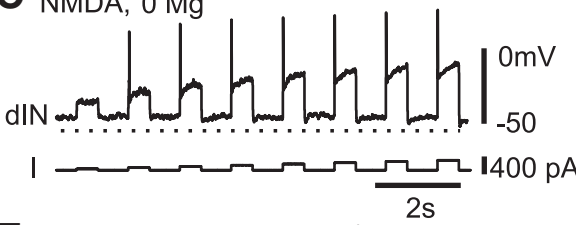

E TTX NMDA, $0 \mathrm{Mg}^{2+}$

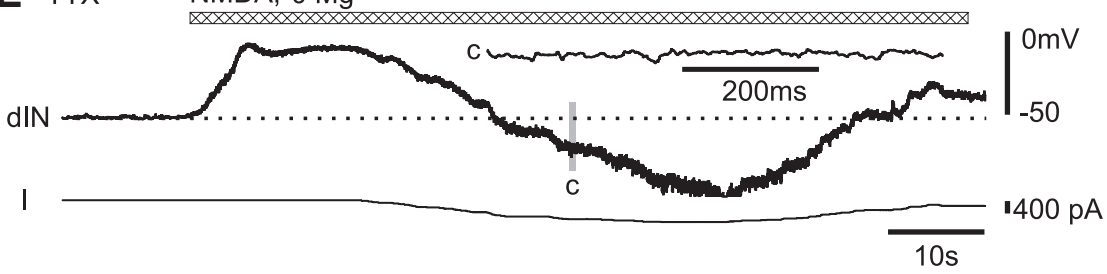

Figure 5. Removal of $\mathrm{Mg}^{2+}$ from the saline abolished both NMDA-induced repetitive firing and TTX-resistant oscillations in dINs. $A$, In saline containing $\mathrm{Mg}^{2+}$, dIN fired repetitively to current injection during NMDA application. $\boldsymbol{B}$, When NMDA leads to larger depolarization, dIN also fires repetitively. C, When $\mathrm{Mg}^{2+}$ was removed from saline, the dIN only fired once to current injection during NMDA application. $D, \ln 0 \mathrm{Mg}^{2+}$, another dIN failed to fire any spikes when depolarized only by NMDA.E, $\ln 0 \mathrm{Mg}^{2+}$ and TTX, NMDA application did not evoke oscillations in a dIN even during negative current injection. $\boldsymbol{a}$-crecords are expanded.
NMDA was applied, the dIN membrane potential depolarized and often passed through a region where oscillations occurred before reaching a depolarized plateau. In these cases, we used negative current injection to bring the membrane potential down to a level at which oscillations were present and stable. Lowering the concentration of NMDA by carefully reducing microperfusion pressure or increasing microperfusion distance (see Materials and Methods) showed that it was not necessary to limit the depolarization by negative current injection for oscillations to be produced ( $n=21 \mathrm{dINs}$ examined).

The TTX-resistant oscillations were seen over a wide range of membrane potentials. Broadly, the peak-to-trough amplitude of the oscillations was biggest at membrane potentials between -25 and $-30 \mathrm{mV}$, when amplitude could reach $>30 \mathrm{mV}$, and became smaller when the membrane potential was either more or less negative than this (Fig. $4 C)(n=6$ dINs). Oscillation frequency changed very little when current injection was used to set membrane potential (Fig. $4 D$ ), typically varying $<1 \mathrm{~Hz}$ over a 100 $\mathrm{mV}$ range (linear regression coefficients: $0.008 \pm 0.015 \mathrm{~Hz} / \mathrm{mV}, n=5 \mathrm{dINs}$ ). This insensitivity of frequency to membrane potential was very similar to that reported for presumed motoneurons by Prime et al. (1999).

The observation that membrane potential oscillations were reliably evoked by NMDA in the presence of TTX suggested that fast sodium channels are not essential for their generation. From a wide range of previous studies, and the membrane potential range at which their amplitude was largest, it seemed very likely that the voltage-dependent unblock and block of NMDARs by $\mathrm{Mg}^{2+}$ could contribute to the oscillations. When $\mathrm{Mg}^{2+}$ was removed from the saline (containing the inhibitory transmitter blockers $1 \mu \mathrm{M}$ strychnine and $20 \mu \mathrm{M}$ SR95531), NMDA application sufficient to produce a small depolarization below dIN firing threshold [around $-28 \mathrm{mV}$ (Sautois et al., 2007)] was no longer able to transform singlefiring dINs to repetitive firing in response to depolarizing current $(n=10)$ (Fig. $5 A, C)$. When the NMDAR depolarization was above dIN firing threshold, dINs in $0 \mathrm{Mg}^{2+}$ saline also failed to fire repetitively in contrast to those in saline with 1 $\mathrm{mM} \mathrm{Mg}^{2+}(n=9)$ (Fig. 5B,D). When TTX was present, NMDA application in 0 $\mathrm{Mg}^{2+}$ saline depolarized dINs but no longer produced oscillations of mem- 
brane potential $(n=6)$ despite varying the membrane potential over a wide range using current injection (Fig. 5E). These observations suggested that the voltagedependent $\mathrm{Mg}^{2+}$ block of NMDARs plays a critical role in the generation of NMDAinduced oscillations and pacemaker firing in dINs.

To determine whether other spinal neurons that are active during swimming showed similar TTX-resistant NMDAinduced oscillations, we examined the activity of 31 non-dIN neurons (13 motoneurons and 18 non-dIN interneurons) after the microperfusion of $100 \mu \mathrm{M}$ NMDA in the presence of TTX. To produce comparable activation of receptors in different cell types, we adjusted the perfusion of NMDA to produce similar levels of depolarization. Generally, closer and higher pressure NMDA perfusion was needed in the non-dINs than in dINs. Some of these neurons (seven motoneurons and four non-dIN interneurons) responded with a sustained depolarization and an increasingly noisy membrane potential but no sign of oscillations (Fig. 6A). The remaining 20 neurons showed some form of oscillatory response (Fig. 6B,C). However, their oscillatory responses appeared to differ significantly from those in dINs and so the two were compared in detail.

To compare NMDA-induced oscillations, autocorrelation analyses were applied to activity from $21 \mathrm{dINs}$ and the 20 other spinal neurons showing some oscillatory response. We measured the following: the main oscillation period $(P)$ and converted this to frequency; the stability of the oscillation (the difference between the first autocorrelation peak and trough) (Fig. 6D); and mean oscillation amplitude. Cluster analyses using the three measures resolved three groups of neuron (Fig. 6E). Twenty-one dINs formed a single cluster characterized by $\sim 10 \mathrm{~Hz}$ oscillations of high amplitude $(9.6 \pm 2.1 \mathrm{~Hz}$; $34.0 \pm 9.4 \mathrm{mV})$. This cluster also contained a single motoneuron, although the oscillations in this motoneuron were rather slower and weaker than most of the dINs in the same cluster $(6.9 \mathrm{~Hz} ; 7.2 \mathrm{mV})$. A second cluster contained two motoneurons and six interneurons and was characterized by a much lower oscillation frequency than the dINs but a high amplitude $(2.2 \pm 1.1 \mathrm{~Hz} ; 27.4 \pm 10.0 \mathrm{mV})$ (Fig. 6B). A third cluster containing three motoneurons and seven interneurons had relatively weak $\sim 5 \mathrm{~Hz}$ oscillations $(5.2 \pm$ $2.6 \mathrm{~Hz} ; 6.2 \pm 4.3 \mathrm{mV}$ ) (Fig. $6 \mathrm{C}$ ). A single interneuron with unreliable oscillations at much higher frequency $(47.5 \mathrm{~Hz} ; 29 \mathrm{mV})$ was close to the dIN cluster but formed a lone dendrogram branch (Fig. 6E, asterisk). The oscillations shown by the dIN group (including the single motoneuron) were significantly different in most measured parameters from the other two groups (Fig. $6 \mathrm{~F}$ ).

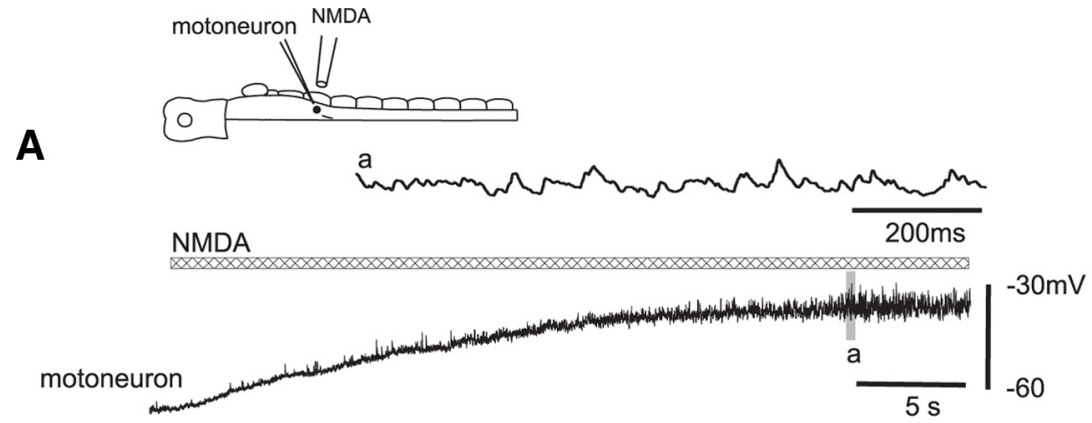

B

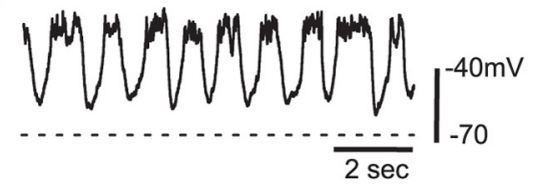

C

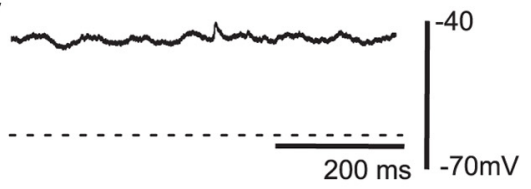

D
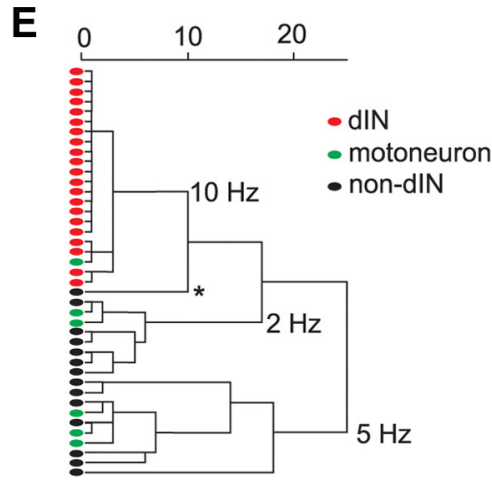

F

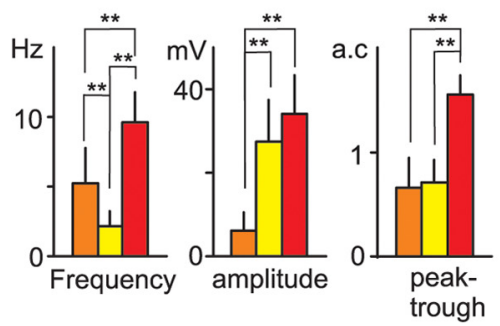

G \%

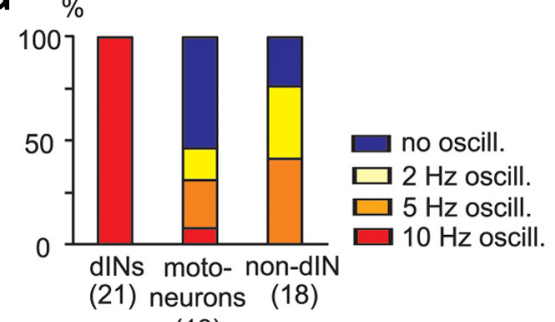

(13)

Figure 6. Comparison of responses of dINs and non-dIN neurons active during swimming to NMDA in TTX.A, A motoneuron only produced depolarization and increased noise. $\boldsymbol{B}$, A non-dIN interneuron produced big, slow oscillations during NMDA-evoked depolarization. $\boldsymbol{C}$, In another motoneuron, there were only small $\sim 10 \mathrm{~Hz}$ oscillations. The dashed lines indicate resting membrane for cluster analysis in $\boldsymbol{E}$. $\boldsymbol{E}$, Dendrogram of a cluster analysis result showing three clusters of neurons with oscillations at 10,2, and Hz. The asterisk $\left(^{*}\right)$ marks the one non-dIN interneuron with high-frequency unreliable big oscillations. $\boldsymbol{F}$, Comparing the oscillation frequencies, amplitudes and autocorrelation peak-to-trough differences in the three clusters in $\boldsymbol{E}\left({ }^{* *} p<0.01\right.$, ANOVA). $\boldsymbol{G}$, The proportion of dINs, motoneurons, and non-dIN interneurons with oscillatory responses. The color coding in $\mathbf{G}$ also applies to $\boldsymbol{F}$. For details, see main text. Error bars indicate SD.

Overall, this analysis shows that reliable, strong $(\sim 10 \mathrm{~Hz})$ membrane potential oscillations in the presence of NMDA are almost exclusively a feature of dINs, and not of other spinal neuron types (summarized in Fig. 6G). At $\sim 10 \mathrm{~Hz}$, these oscillations lie at the lower end of the normal range of swimming frequencies in the hatchling tadpole. The variability of dIN oscillations, reflected in the distances between individual cells of the dIN cluster in the dendrogram, appears to be smaller than for the other two groupings.

\section{Effects of a gap junction blocker on NMDA-induced oscillations}

An important factor to consider is that the dINs form an electrically coupled population. Gap junctions and electrical coupling 
A

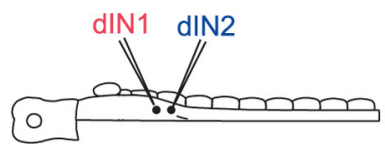

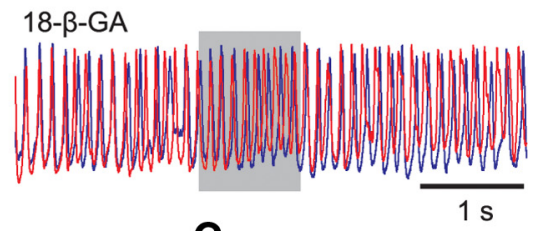

B
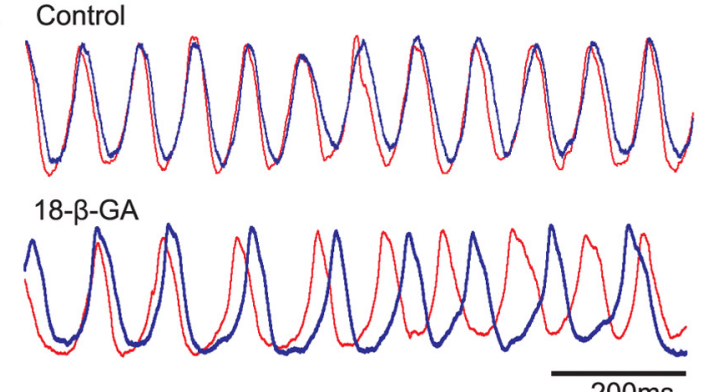

D
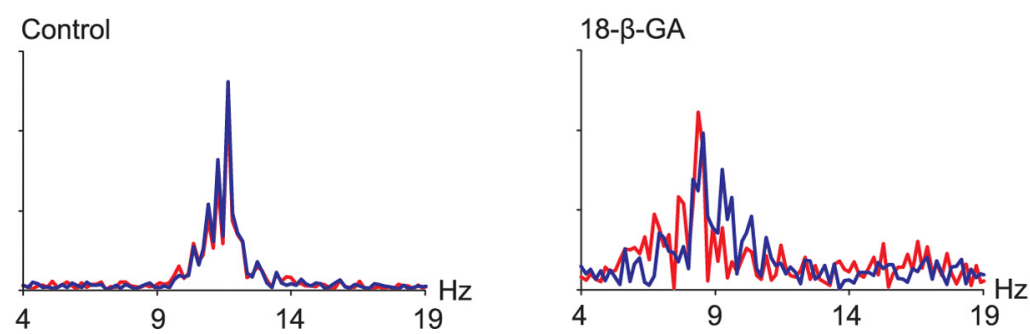

Figure 7. Blocking gap junctions desynchronizes the oscillations evoked by NMDA application in TTX. $A$, Simultaneous recording from two dINs shows their oscillations in control and in 18- $\beta$-GA. B, Desynchronization of NMDA oscillations in $18-\beta$-GA. Stretched 1 s oscillations marked by gray bars in $A$ to show the synchrony of oscillation in the two dINs. Amplitudes of oscillations (blue from $25.4 \mathrm{mV}$ in control to $39.8 \mathrm{mV}$, red from 26.8 to $39.2 \mathrm{mV}$ ) were normalized and records overlapped for easier comparisons in $A$ and B. C, Polar plots of the mean relative phase of oscillations in five pairs of simultaneously recorded dINs before (black arrows) and after 18- $\beta$-GA application (green arrows, $5.5 \mathrm{~s}$ of oscillation analyzed in each record). D, Power spectrum of $5.5 \mathrm{~s}$ of oscillation in control and during the coupling block in $\boldsymbol{A}$.

play a role in neuronal network rhythm generation and membrane potential oscillations in many other systems. We recently reported electrical coupling between dINs in the tadpole (Li et al., 2009). It was therefore important to explore the significance of this electrical coupling in TTX-resistant NMDA-induced oscillations in dINs. In this same recent study, we assessed a range of gap junction blockers in the tadpole and found $40 \mu \mathrm{M} 18-\beta$-GA to provide the best combination of potency and specificity. 18$\beta$-GA was therefore used to block the electrical coupling in five simultaneous recordings from 10 dINs. To maintain long and stable whole-cell recordings, the perforated patch-clamp method was used. Simultaneous recordings allowed us to monitor directly the timing and effectiveness of electrical coupling block. $18-\beta$-GA reduced coupling coefficients from $8 \pm 3.2$ to $1.6 \pm$ $0.3 \%$ ( $78 \%$ block) after application for $\sim 30 \mathrm{~min}$. When the electrical coupling block stabilized, the ability of dINs to generate TTX-resistant $\sim 10 \mathrm{~Hz}$ oscillations to bath-applied NMDA persisted (Fig. 7A). However, synchronization between the simultaneously recorded dIN pairs was significantly weakened in 18- $\beta$-GA (Fig. 7B). Cross-correlation analysis showed that NMDA-induced oscillations were highly regular and synchronized in control (peak cross-correlation coefficients: $0.88 \pm 0.07$, $n=5$ pairs). In $18-\beta$-GA, cross-correlation peak coefficients fell significantly $(0.51 \pm 0.15 ; n=5$; paired $t$ test, $p=0.012)$. This weakening of synchronization is clearly shown in polar plots of the mean phase differences between oscillations in pairs of dINs (Fig. 7C). Power spectrum analysis showed that, with the weakening of synchronization, the range of oscillation frequencies increased while peak frequencies significantly decreased (from $10.6 \pm 2$ to $7.5 \pm 1.3 \mathrm{~Hz} ; n=10$ dINs; paired $t$ test, $p<0.001$ ) (Fig. 7D). These results show that gap junctions play a significant role in synchronizing oscillations between coupled dINs, and presumably across the whole dIN population, and by doing so also increase and stabilize oscillation frequency (Soto-Treviño et al., 2005). There was an increase in oscillation amplitudes after 18- $\beta$-GA block (28.7 \pm 11.4 to $38.8 \pm 6.7 \mathrm{mV}$; paired $t$ test, $p=$ $0.016)$, which could result from electrical coupling block. However, differences in oscillation amplitudes are very sensitive to average membrane potential levels (Fig. 4C) and are also affected by the progress of amphotericin perforation of patch membrane at the beginning of recordings.

\section{Swimming-like rhythms in hemi-CNS preparations}

If the conditional pacemaker properties of dINs induced by NMDA play a role in the generation and maintenance of swimming activity, then there are two predictions: first, swimming-like activity should be present when the inhibition that leads to postinhibitory rebound firing in dINs is blocked (Soffe, 1989); second, swimminglike activity should be blocked by treatments that block NMDA-induced dIN pacemaker properties (NMDAR antagonists and $0 \mathrm{Mg}^{2+}$ saline). To test these predictions in hemi-CNS preparations, we used $20 \mu \mathrm{M}$ SR95531 and $1 \mu \mathrm{M}$ strychnine together to block GABA and glycine inhibition. To initiate swimming-like motor activity without application of NMDA, three or four $1 \mathrm{~ms}$ current pulses at $30-40 \mathrm{~Hz}$ were applied to the hindbrain region (approximately seventh rhombomere region). These stimuli evoked sustained rhythmic motor nerve bursts in all 10 hemi-CNS preparations tested (Fig. 8 A,C) (mean episode length, $5.3 \pm 3.7 \mathrm{~s}$; range, $1.4-21.1 \mathrm{~s}$ ). These episodes were shorter than fictive swimming episodes seen in intact tadpoles. The frequency of motor nerve bursts fell quickly at the start of each episode (Fig. $8 E$ ). The average frequency was $20.6 \pm 0.8 \mathrm{~Hz}(5$ tadpoles, 17 episodes), similar to that seen in the first $5 \mathrm{~s}$ in intact tadpole fictive swimming $(19.1 \pm 1.8 \mathrm{~Hz} ; n=6$ tadpoles; 15 episodes; $p=0.21$ ) (Fig. $8 F$ ).

When $25 \mu \mathrm{M}$ of the NMDAR antagonist D-AP5 was bathapplied for $5 \mathrm{~min}$, these episodes of rhythmic motor nerve activity were shortened by $98.4 \pm 2.4 \%$ (mean episode length: $0.12 \pm$ $0.19 \mathrm{~s} ; p=0.002$, paired $t$ test; $n=5$ tadpoles) (Fig. $8 A, B$ ). There was partial recovery after washing for 15 min (mean episode length: $2.0 \pm 2.3 \mathrm{~s} ; 29 \pm 35.6 \%$ ). Critically, the rhythmic activity was also significantly shortened by $90.6 \pm 15.1 \%$ when tadpoles were bathed in saline with $0 \mathrm{Mg}^{2+}$ (mean episode length: $0.38 \pm$ $0.39 \mathrm{~s} ; p=0.005$, paired $t$ test; $n=5$ tadpoles) (Fig. $8 C, D$ ) with partial recovery after washing for $10 \mathrm{~min}(63.9 \pm 48.5 \%)$. These 
data suggest that both NMDAR activation and $\mathrm{Mg}^{2+}$ are important in the generation of rhythmic activity in the simplified hemi-CNS preparation in the presence of inhibitory transmitter blockers.

\section{NMDA induced hemi-CNS rhythmicity} Direct recordings previously showed that, when active, dINs release glutamate to excite each other (Li et al., 2006). The above results have now shown that NMDAR activation by glutamate or NMDA can modulate the firing properties of these dINs, making them able to fire repetitively at frequencies similar to rhythmic swimming and therefore to act as conditional pacemakers. In addition, our extracellular recordings from intact spinal cord and hemi-CNS preparations showed that swimming-like rhythmic activity, after removing inhibition, depended on NMDAR activation. To test our conclusion that the conditional pacemaker activity of dINs provides a mechanism for rhythm generation, we checked that direct microperfusion of NMDA onto a hemi-CNS preparation led to rhythm generation even when inhibition was blocked. This also allowed us to directly examine the activity of dINs during rhythmic activity and test the quality of the rhythm generated. One hundred micromolar NMDA was microperfused onto the caudal hindbrain with inhibitory transmitter blockers ( $1 \mu \mathrm{M}$ strychnine and $20 \mu \mathrm{M}$ SR95531) in the saline. A suction electrode was placed on the caudal spinal cord to record spinal network activity. NMDA application resulted in reliable rhythmic activity, which, unlike the responses to electrical stimulation described above (Fig. 8A,C), could persist as long as NMDA was present (tested up to $260 \mathrm{~s}$ ) in 13 of 13 tadpoles (>300 trials) (Fig. 9A,C).

Whole-cell recordings were made from nine dINs during NMDA-induced hemi-CNS rhythmic activity. All these dINs were depolarized and fired reliably throughout rhythmic network activity. However, very often (108 of 162 trials), rhythmic dIN firing began before the hemi-CNS rhythm, consistent with its initiating the network rhythm. During hemi-CNS rhythmic activity, dINs reliably fired a single spike in phase with each rhythmic spinal cord (s.c.) burst (Fig. 9A). In contrast, recordings from 22 non-dIN interneurons showed a different form of response in that most (7 of 7 motoneurons; 11 of 15 non-dINs) responded to microperfused NMDA with a relatively weak depolarization $(6 \pm 3.89 \mathrm{mV}$; range, $0-15 \mathrm{mV})$ and never fired until the whole spinal cord network had become active ( $n=191$ trials) (Fig. 9C). The remaining four non-dINs belonging to the two types of inhibitory interneuron in the swimming circuit sometimes (14 in 26 trials) responded to NMDA with a stronger depolarization and occasional firing $(5-38 \mathrm{~Hz})$ (not illustrated).

The whole-cell recordings, which suggest that firing occurs exclusively in dINs before the onset of hemi-CNS rhythmic activity and show that they fire reliably throughout rhythmic activ-
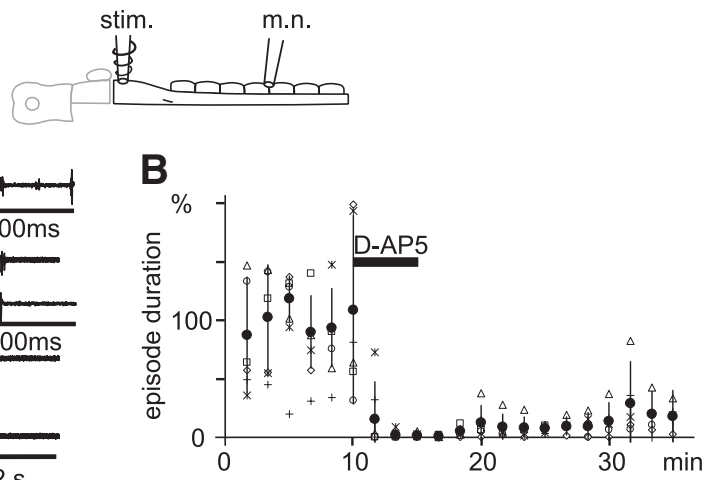

D

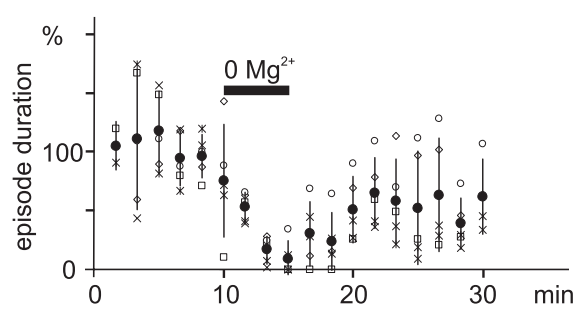

$\mathbf{F}$

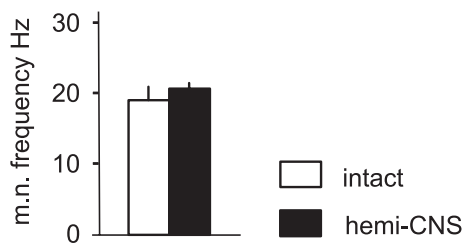

Figure 8. Endogenous swimming-like activity in hemi-CNS without inhibition relies on NMDAR activation and $\mathrm{Mg}^{2+} . A, A n$ example of motor nerve (m.n.) records showing swimming-like activity episodes before, during, and after D-AP5 application (25 $\mu \mathrm{m} ; 5 \mathrm{~min}) . \boldsymbol{B}$, Time series plots showing changes in episode durations in five experiments when hindbrain was stimulated every timuli at time $=0$. The gray symbols are from 17 episodes in five tadpoles, and the black dots are moving averages. $\boldsymbol{F}$, The hemi-CNS m.n. rhythm frequency is similar to that in the first $5 \mathrm{~s}$ of that in intact tadpole fictive swimming. In $\boldsymbol{A}$ and $\boldsymbol{C}$, insets $\boldsymbol{a}-\boldsymbol{C}$ show m.n. at expanded timescale. Error bars indicate SD.

ity, again focused attention on the central role of the dINs. If conditional pacemaker activity in dINs was driving the hemiCNS rhythm, we might expect that the quality of rhythm (stability and consistency) would be stronger in the dINs than in other neurons. However, we might expect the quality to be less strong than in the intact tadpole with inhibition and therefore postinhibitory rebound firing present. To address these questions, we used the coefficient of rhythmicity (see Materials and Methods) to assess the quality of fictive swimming generated after sensory stimulation in the intact tadpole and the swimming-like rhythms in the hemi-CNS in response to NMDA. This comparison appears reasonable given that the frequencies of spinal cord rhythms in the hemi-CNS induced by NMDA microperfusion $(21.1 \pm 3.3 \mathrm{~Hz} ; n=7$ tadpoles) were very similar to those in the first $5 \mathrm{~s}$ of fictive swimming in intact tadpoles without inhibitory transmitter blockers $(p=0.73)$.

We first compared the coefficient of rhythmicity $(\mathrm{Cr})$ of spikes in individual whole-cell recordings and $\mathrm{Cr}$ of motor nerve bursts (monitoring motor output) in intact tadpoles. These were then compared with similar measurements in hemi-CNSs without inhibition. The value of $\mathrm{Cr}$ for dIN firing in the intact tadpole was very high $(1.00 ; n=6)$. Values for non-dINs and for motor nerve activity in the intact animals were almost as high $(0.94 \pm 0.07$ and 


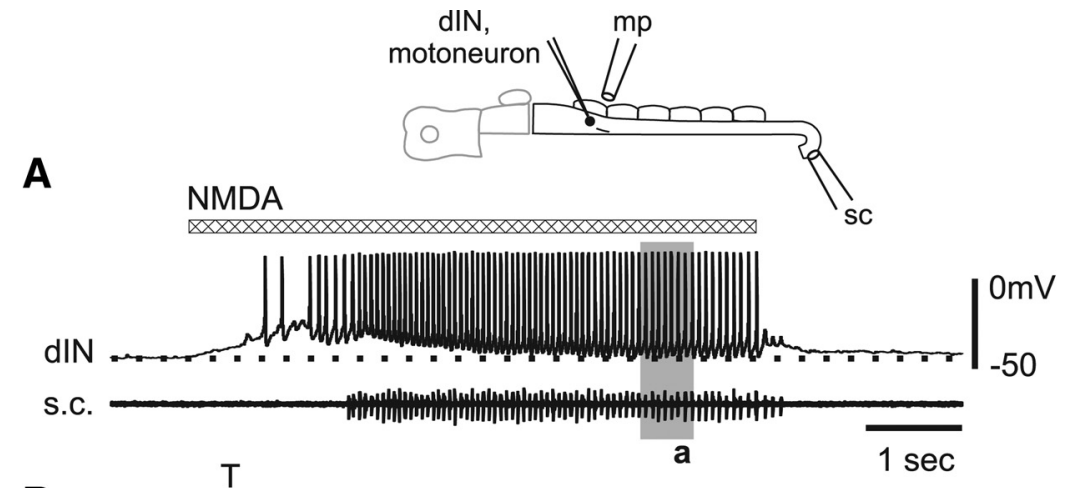

B

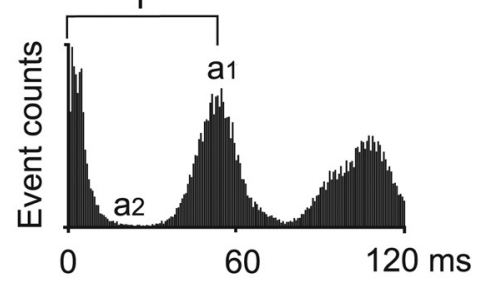

C

NMDA
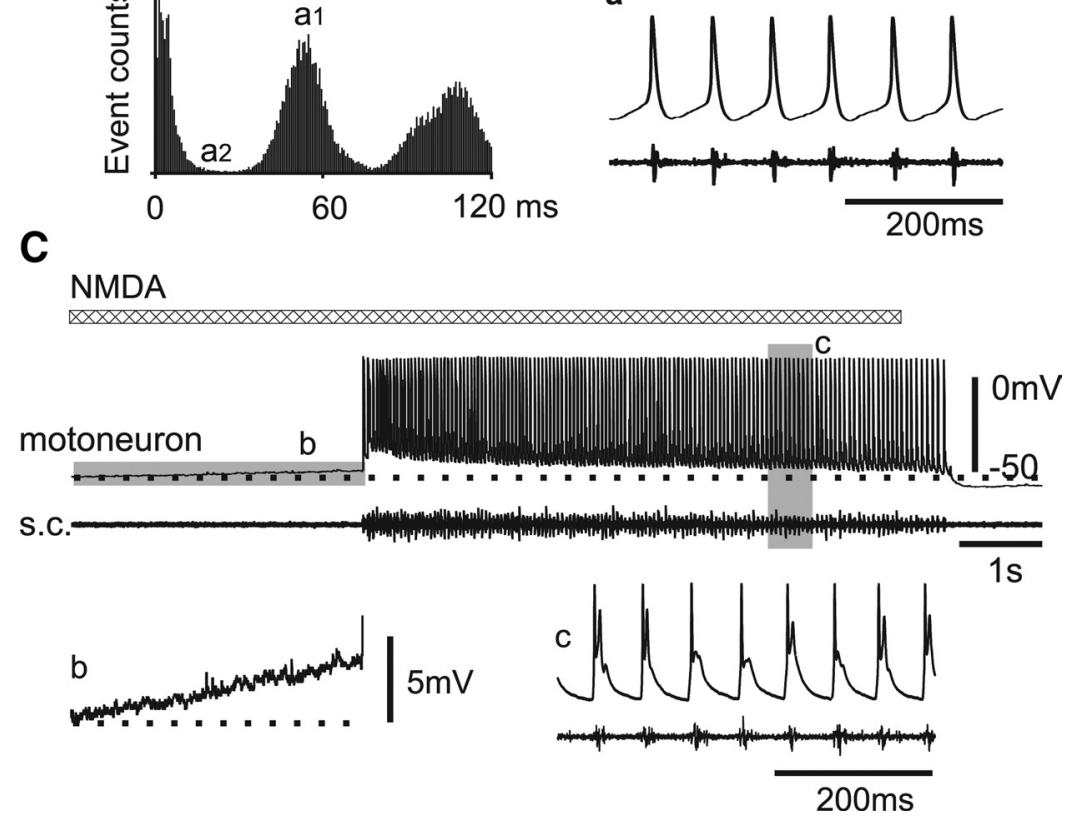

Figure 9. Network activity in hemi-CNS induced by local microperfusion of NMDA onto the hindbrain in saline containing $1 \mu \mathrm{M}$ strychnine and $20 \mu \mathrm{m}$ SR95531. A, dIN is depolarized and starts to fire before rhythmic s.c. activity starts. $\boldsymbol{B}$, Distribution histogram of the intervals between s.c. burst triggered events in $\boldsymbol{A}$. a1 and a2 are peak and trough counts respectively used for calculating coefficient of rhythmicity ( $\mathrm{Cr}=0.99$ for record in $\boldsymbol{A})$. $T$ is used for calculating rhythm frequency. $\boldsymbol{C}$, A motoneuron is only slightly depolarized and starts to fire when rhythmic s.c. activity starts.

$0.98 \pm 0.01 ; n=6$ tadpoles analyzed). Surprisingly, values of $\mathrm{Cr}$ for dIN firing in hemi-CNS rhythms induced by NMDA were also very high, even in the presence of inhibitory transmitter blockers and therefore in the absence of postinhibitory rebound $(1.0 ; n=6)$. In contrast, the values for non-dINs and for spinal cord bursts (monitoring network activity) in the hemi-CNS were significantly lower $(0.61 \pm 0.16, n=18$ non-dINs; and $0.70 \pm$ $0.16, n=7$ tadpoles; see above; $p<0.01$ ). This reduction in the quality of the motor rhythm compared with the firing of dINs was not surprising given that the blockers were likely to have removed precise inhibitory timing information.

Overall, these results together with those of the previous section indicate that, in the presence of normal extracellular $\mathrm{Mg}^{2+}$ and with inhibition removed, NMDAR activation alone can be sufficient to induce and maintain rhythmic activity in dINs, which drives rhythmic activity in the whole spinal cord motor network.

NMDA-induced conditional pacemaker activity of dINs not only appears to provide an additional mechanism for rhythm generation within each side of the CNS, and not requiring inhibition, but it can produce rhythmic activity equivalent to that seen during swimming in the intact animal. It should therefore be possible to simply remove inhibition from an intact CNS and reveal independent rhythms on the two sides. To test this, we applied $30-50 \mu \mathrm{m}$ NMDA to a $\sim 700 \mu \mathrm{m}$ length of spinal cord isolated by transecting at the third and seventh post otic segments and removed inhibition by using $1 \mu \mathrm{M}$ strychnine and $10-20 \mu \mathrm{M}$ SR95531 or $20 \mu \mathrm{M}$ bicuculline (Fig. 10A, diagram). Soon after introducing the inhibitory transmitter blockers, NMDA application induced rhythmic swimming activity, which alternated between the two sides (mean frequency, $22.9 \pm 5.2 \mathrm{~Hz} ; n=5$ animals) (Fig. 10A,B). Left-right coupling then became lost indicating a significant reduction of inhibition between the two sides. Despite this, rhythmic activity continued independently on each side at similar frequencies (right side, $22.4 \pm 3.1 \mathrm{~Hz}$; left side, $22.8 \pm 3.7 \mathrm{~Hz}$ ) (Fig. 10C,D). These independent rhythms were presumably not attributable to postinhibitory rebound but were most plausibly attributable to conditional pacemaker activity in spinal cord members of the dIN populations on each side of the CNS. In support of this, it has been shown previously that little rhythm can be generated in the spinal cord by applied NMDA when in $0 \mathrm{~mm}$ $\mathrm{Mg}^{2+}$ (Soffe and Roberts, 1989).

\section{Discussion}

This paper describes a novel type of ionotropic receptor-mediated self-resetting within a defined population of brainstem neurons. Intrinsic modulation of neuronal and synaptic network properties by other network members occurs in invertebrates (Katz and Frost, 1996; Sakurai and Katz, 2003), mediated by neuromodulators such as serotonin or peptides, and can be state dependent, altering according to presynaptic and postsynaptic activity. Intrinsic modulation has also been described in vertebrates, primarily involving metabotropic transmitter receptors [e.g., metabotropic glutamate receptors (El Manira et al., 2002; Lieske and Ramirez, 2006; Chapman et al., 2008)]. However, here we describe a self-resetting role for ionotropic NMDA receptors. We have shown that the agonist NMDA, or the likely transmitter L-glutamate, can transform the reticulospinal dIN neurons that drive tadpole swimming ( $\mathrm{Li}$ et al., 2006; Soffe et al., 2009) into pacemakers that fire repetitively within the swimming frequency range. With spiking blocked, NMDA application produces oscillations in these neurons at similar frequencies. Since dINs synapse with each other and release glutamate to activate NMDARs (Li et al., 2006), they can modulate the firing properties of their own population, specifically while in an active state. This targeted self-resetting allows one particular neuron type, the excitatory dIN neurons driving swimming, to be transformed rapidly (equivalent to a few swimming cycles) into pacemaker mode only while rhythm generation is occurring. One consequence is that 
A
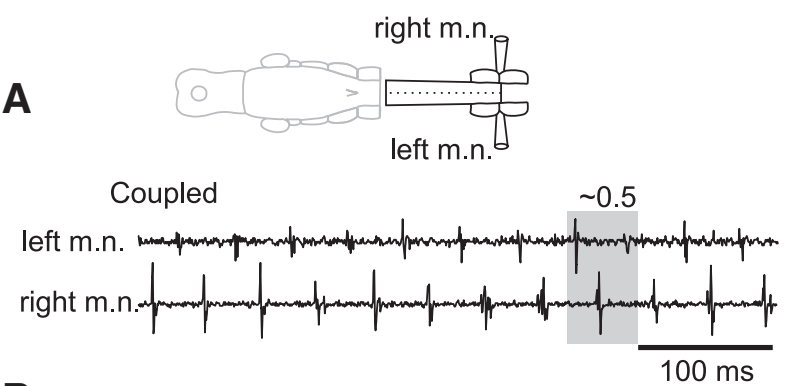

B

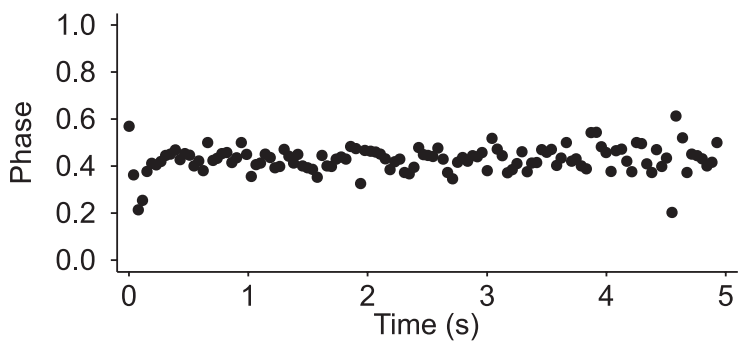

C

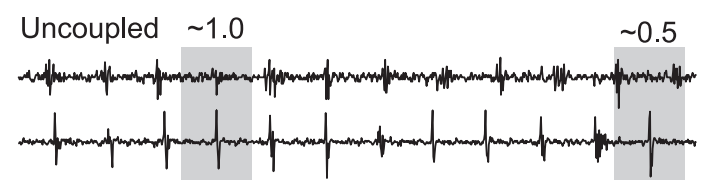

D

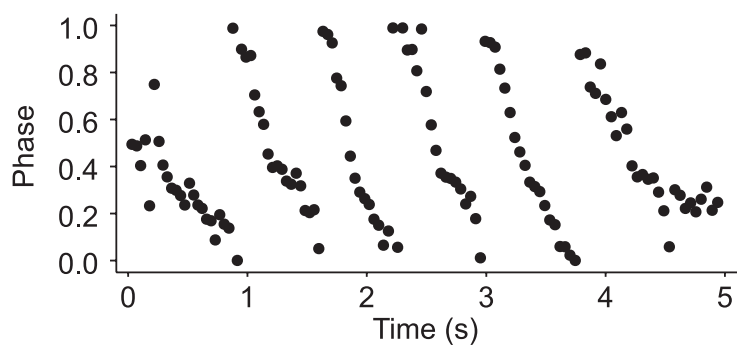

Figure 10. Network activity in the spinal cord induced by bath perfusion of NMDA. $\boldsymbol{A}$, Sustained swimming rhythm recorded from motor nerves (m.n.) on opposite sides of a $\sim 700 \mu \mathrm{m}$ length of spinal cord. $\boldsymbol{B}$, Phase plot showing activity alternates between the two sides (also see shaded cycle in $\boldsymbol{A}$ ). $\boldsymbol{C}$, Rhythm continues despite phase uncoupling produced by $1 \mu \mathrm{m}$ strychnine and $20 \mu \mathrm{m}$ SR95531. D, Phase plot for record in $\boldsymbol{C}$.

the pacemaker properties of neurons during activity are not revealed when studied at rest.

Many rhythm-generating networks controlling movement comprise symmetrical half-centers coupled by reciprocal inhibition (Arshavsky et al., 1993; Marder et al., 2005; Feldman and Del Negro, 2006; Marder and Bucher, 2007; Brocard et al., 2010). Inhibition may play a role in rhythm generation, but many networks can operate without it (Droge and Tao, 1993; Cowley and Schmidt, 1995; Ozaki et al., 1996; Kremer and Lev-Tov, 1997; Rioult-Pedotti, 1997; Stein et al., 1998; Cui et al., 2004, 2005; Cangiano and Grillner, 2005; Samara and Currie, 2008). The new self-resetting mechanism we describe provides one mechanism to explain this. The simple central pattern generator (CPG) network for tadpole swimming only contains dINs, two types of glycinergic inhibitory interneurons and motoneurons, greatly facilitating analysis of rhythm generation (Roberts et al., 2008). The current "emergent network" hypothesis is based on network connections in which dINs feed back glutamate excitation, depolarize each other by activating NMDARs, and then fire on rebound after reciprocal inhibition from the other side of the CNS (Li et al., 2006; Roberts et al., 2008; Soffe et al., 2009). With inhibition blocked pharmacologically and by removing one side of the CNS, some rhythm remained (Kahn and Roberts, 1982; Soffe, 1989).
This suggested that pacemaker neurons were present but they remained elusive, even though the hemi-CNS with inhibition blocked simplified the CPG network to just dINs and motoneurons. Based on in situ whole-cell recordings, Xenopus tadpole swimming CPG neurons were proposed to be conditional oscillators because they could fire repetitively (Aiken et al., 2003). However, our much larger sample of recordings showed that most CPG neurons either fire repetitively at much higher frequencies than the normal swimming range (Sautois et al., 2007) or singly (dINs) to current injections (Li et al., 2006). Little evidence supported the conditional oscillator proposal. Our present results show that dINs are transformed by NMDAR activation into pacemakers, firing at frequencies seen during swimming. In contrast, NMDA application has little effect on the highfrequency firing of other spinal CPG interneurons. Because only dINs fire at swimming frequencies during NMDAR activation and because they provide the excitatory input onto motoneurons, they are the clear candidates to drive hemi-CNS rhythms after either NMDA application or hindbrain stimulation. The hatchling Xenopus tadpole swimming CPG therefore uses a curious new mechanism, self-resetting, to allow rhythm generation without inhibition.

What is the role of NMDAR-dependent oscillations in rhythmic systems? The oscillations we have described in tadpole dINs, in TTX, could directly drive their NMDAR-induced pacemaker firing, as suggested by their similar $(\sim 10 \mathrm{~Hz})$ frequencies. However, it is more likely that the currents responsible for the oscillations normally interact with fast spike currents and fast ligand-gated synaptic currents in a more complex way to influence dIN rhythmic firing during swimming, perhaps stabilizing and constraining the frequency range. Since dINs appear to be the only tadpole spinal neurons showing fast, NMDAinduced oscillations [those described from presumed motoneurons by Prime et al. (1999) may actually have been from dINs], it should be possible to discover their role. This is important given the compelling evidence that hindbrain and spinal dINs provide the synaptic excitation that drives tadpole swimming ( $\mathrm{Li}$ et al., 2006; Soffe et al., 2009). The functional significance of the much slower $(\leq 1 \mathrm{~Hz})$ NMDA-induced, TTX-resistant oscillations reported in other animals, from neurons including spinal motoneurons and some interneurons, remains unclear. In motoneurons of lamprey, frog tadpoles, and adult frogs (Grillner and Wallén, 1985; Wallén and Grillner, 1985, 1987; Sillar and Simmers, 1994; Rioult-Pedotti, 1997; Reith and Sillar, 1998; Prime et al., 1999), oscillation frequencies are somewhat slower than the locomotor rhythm. In motoneurons of turtles (Guertin and Hounsgaard, 1998) and mammals (Hochman et al., 1994b; MacLean et al., 1997; Tresch and Kiehn, 2000), oscillation frequencies are closer to those during fictive locomotion. However, it is uncertain whether the motoneurons are necessary for rhythm generation (Cowley et al., 2005), since motoneurons are not generally considered part of vertebrate CPGs, or whether the oscillatory mechanisms help pattern motoneuron firing (Brocard et al., 2010). In neonatal rat, slow $(\leq 1 \mathrm{~Hz})$ TTX-resistant, NMDAinduced oscillations are seen in interneurons lying around the central canal (Hochman et al., 1994a). Some of them may be counterparts to $\mathrm{Hb} 9$-positive glutamatergic neurons in mice (Wilson et al., 2005; Ziskind-Conhaim et al., 2008), which are possible components of the spinal rhythm-generating network (Brownstone and Wilson, 2008; Kwan et al., 2009; Brocard et al., 2010). Faster TTX-resistant, NMDA-induced oscillations seem less common but are found in some interneurons surrounding the neonatal rat central canal (Hochman et al., 1994a), as well as 
some adult Xenopus spinal motoneurons (Rioult-Pedotti, 1997), hippocampal pyramidal cells (Ganong and Cotman, 1986), and midbrain dopaminergic neurons (Deister et al., 2009). However, their roles are not known.

In this study we have shown that $\mathrm{Mg}^{2+}$ is necessary for the NMDA-induced, TTX-resistant dIN oscillations, repetitive dIN firing, and hemi-CNS rhythms when inhibition was blocked. However, without $\mathrm{Mg}^{2+}$ dINs do not fire to slow depolarizations and only fire once at the onset of depolarizing current injections even when NMDA is present. Experiments in TTX have shown that $\mathrm{Mg}^{2+}$ in some way promotes the generation of membrane potential oscillations, presumably by voltage-dependent blocking and unblocking of NMDARs but the role of other currents (like $\mathrm{Ca}^{2+}$-dependent $\mathrm{K}^{+}$) is not yet clear. When dINs act as pacemakers to drive hemi-CNS rhythms, similar mechanisms are presumably operating but remain to be investigated. The role for $\mathrm{Mg}^{2+}$ here may be more complicated because $\mathrm{Mg}^{2+}$ can also block other currents like L-type calcium currents (Kuo and Hess, 1993). In lamprey, it has been proposed that $\mathrm{Ca}^{2+}$-dependent $\mathrm{K}^{+}$currents, low-voltage-activated $\mathrm{Ca}^{2+}$ currents and $\mathrm{Na}^{+}{ }_{-}$ dependent $\mathrm{K}^{+}$currents could interact with TTX-resistant NMDA oscillations to mediate undulating motoneuron membrane potential changes during fictive swimming (for review, see Grillner, 2003). Investigation of such currents in Xenopus dINs is hampered by their mutual electrical coupling (Li et al., 2009) and the lack of satisfactory, coupling blockers. Electrical coupling is a feature of many other rhythm-generating networks, and there is debate about whether it contributes to rhythm generation (for review, see Kiehn and Tresch, 2002; Connors and Long, 2004). In the tadpole, significant block of electrical coupling desynchronizes but does not stop NMDA-induced oscillations, although it makes the normal swimming rhythm less reliable (Li et al., 2009). The coupling makes direct investigation of the cellular mechanisms of oscillations difficult. For example, hyperpolarizing a single dIN does not change oscillation frequency or block oscillations as it should in an uncoupled neuron (Fig. 4C,D). If repolarization depends on $\mathrm{Ca}^{2+}$-dependent $\mathrm{K}^{+}$currents, then intracellular BAPTA injection might prevent it but oscillations can still spread in from other dINs. Transformation of dINs into pacemakers by NMDAR activation requires an inward current activated as the partial $\mathrm{Mg}^{2+}$ block becomes reduced by depolarization, and then a repolarization process after the spike. Other currents may contribute to pacemaker properties, for example, the persistent sodium current $\left(I_{\mathrm{NaP}}\right)$ identified in mammalian locomotor CPG neurons (Tazerart et al., 2008). All of this remains to be investigated.

In conclusion, our evidence shows that NMDA-induced $10-25 \mathrm{~Hz}$ repetitive firing of excitatory reticulospinal neurons (dINs), in the presence of $\mathrm{Mg}^{2+}$, can generate a swimming-like rhythm in both the half- and intact CNS when inhibition is blocked. This rapid intrinsic self-resetting acts exclusively between members of a single homogeneous population of excitatory premotor interneurons and is mediated by an ionotropic transmitter receptor. It may give the rhythm-generating network additional robustness in life in which many such networks have multiple rhythm generation mechanisms (Marder et al., 2005).

\section{References}

Aiken SP, Kuenzi FM, Dale N (2003) Xenopus embryonic spinal neurons recorded in situ with patch-clamp electrodes-conditional oscillators after all? Eur J Neurosci 18:333-343.

Arshavsky YI (2003) Cellular and network properties in the functioning of the nervous system: from central pattern generators to cognition. Brain Res Brain Res Rev 41:229-267.
Arshavsky YuI, Gelfand IM, Orlovsky GN, Pavlova GA, Popova LB (1984) Origin of signals conveyed by the ventral spino-cerebellar tract and spinoreticulo-cerebellar pathway. Exp Brain Res 54:426-431.

Arshavsky YuI, Orlovsky GN, Panchin YuV, Roberts A, Soffe SR (1993) Neuronal control of swimming locomotion: analysis of the pteropod mollusc Clione and embryos of the amphibian Xenopus. Trends Neurosci 16:227-233.

Atsuta Y, Garcia-Rill E, Skinner RD (1990) Characteristics of electrically induced locomotion in rat in vitro brain stem-spinal cord preparation. J Neurophysiol 64:727-735.

Brocard F, Tazerart S, Vinay L (2010) Do pacemakers drive the central pattern generator for locomotion in mammals? Neuroscientist 16:139-155.

Brownstone RM, Wilson JM (2008) Strategies for delineating spinal locomotor rhythm-generating networks and the possible role of $\mathrm{Hb} 9$ interneurones in rhythmogenesis. Brain Res Rev 57:64-76.

Buzsáki G, Draguhn A (2004) Neuronal oscillations in cortical networks. Science 304:1926-1929.

Cangiano L, Grillner S (2003) Fast and slow locomotor burst generation in the hemispinal cord of the lamprey. J Neurophysiol 89:2931-2942.

Cangiano L, Grillner S (2005) Mechanisms of rhythm generation in a spinal locomotor network deprived of crossed connections: the lamprey hemicord. J Neurosci 25:923-935.

Chapman RJ, Issberner JP, Sillar KT (2008) Group I mGluRs increase locomotor network excitability in Xenopus tadpoles via presynaptic inhibition of glycinergic neurotransmission. Eur J Neurosci 28:903-913.

Connors BW, Long MA (2004) Electrical synapses in the mammalian brain. Annu Rev Neurosci 27:393-418.

Cowley KC, Schmidt BJ (1995) Effects of inhibitory amino acid antagonists on reciprocal inhibitory interactions during rhythmic motor activity in the in vitro neonatal rat spinal cord. J Neurophysiol 74:1109-1117.

Cowley KC, Zaporozhets E, Maclean JN, Schmidt BJ (2005) Is NMDA receptor activation essential for the production of locomotor-like activity in the neonatal rat spinal cord? J Neurophysiol 94:3805-3814.

Cui WW, Saint-Amant L, Kuwada JY (2004) shocked gene is required for the function of a premotor network in the zebrafish CNS. J Neurophysiol 92:2898-2908.

Cui WW, Low SE, Hirata H, Saint-Amant L, Geisler R, Hume RI, Kuwada JY (2005) The zebrafish shocked gene encodes a glycine transporter and is essential for the function of early neural circuits in the CNS. J Neurosci 25:6610-6620.

Deister CA, Teagarden MA, Wilson CJ, Paladini CA (2009) An intrinsic neuronal oscillator underlies dopaminergic neuron bursting. J Neurosci 29:15888-15897.

Droge MH, Tao Y (1993) Glycine effects on in vitro motor pattern generation in mouse spinal cord. Neurosci Lett 158:139-142.

El Manira A, Kettunen P, Hess D, Krieger P (2002) Metabotropic glutamate receptors provide intrinsic modulation of the lamprey locomotor network. Brain Res Brain Res Rev 40:9-18.

Feldman JL, Del Negro CA (2006) Looking for inspiration: new perspectives on respiratory rhythm. Nat Rev Neurosci 7:232-242.

Ganong AH, Cotman CW (1986) Kynurenic acid and quinolinic acid act at $\mathrm{N}$-methyl-D-aspartate receptors in the rat hippocampus. J Pharmacol Exp Ther 236:293-299.

Getting PA (1989) Emerging principles governing the operation of neural networks. Annu Rev Neurosci 12:185-204.

Grillner S (2003) The motor infrastructure: from ion channels to neuronal networks. Nat Rev Neurosci 4:573-586.

Grillner S, Wallén P (1985) The ionic mechanisms underlying N-methyl-Daspartate receptor-induced, tetrodotoxin-resistant membrane potential oscillations in lamprey neurons active during locomotion. Neurosci Lett 60:289-294.

Guertin PA (2009) The mammalian central pattern generator for locomotion. Brain Res Rev 62:45-56.

Guertin PA, Hounsgaard J (1998) NMDA-induced intrinsic voltage oscillations depend on L-type calcium channels in spinal motoneurons of adult turtles. J Neurophysiol 80:3380-3382.

Harris-Warrick RM (2002) Voltage-sensitive ion channels in rhythmic motor systems. Curr Opin Neurobiol 12:646-651.

Hochman S, Jordan LM, MacDonald JF (1994a) N-Methyl-D-aspartate receptor-mediated voltage oscillations in neurons surrounding the central canal in slices of rat spinal cord. J Neurophysiol 72:565-577.

Hochman S, Jordan LM, Schmidt BJ (1994b) TTX-resistant NMDA 
receptor-mediated voltage oscillations in mammalian lumbar motoneurons. J Neurophysiol 72:2559-2562.

Kahn JA, Roberts A (1982) Experiments on the central pattern generator for swimming in amphibian embryos. Philos Trans R Soc Lond B Biol Sci 296:229-243.

Kahn JA, Roberts A, Kashin SM (1982) The neuromuscular basis of swimming movements in embryos of the amphibian Xenopus laevis. J Exp Biol 99:175-184.

Katz PS, Frost WN (1996) Intrinsic neuromodulation: altering neuronal circuits from within. Trends Neurosci 19:54-61.

Kiehn O, Tresch MC (2002) Gap junctions and motor behavior. Trends Neurosci 25:108-115.

Kremer E, Lev-Tov A (1997) Localization of the spinal network associated with generation of hindlimb locomotion in the neonatal rat and organization of its transverse coupling system. J Neurophysiol 77:1155-1170.

Kuo CC, Hess P (1993) Block of the L-type $\mathrm{Ca}^{2+}$ channel pore by external and internal $\mathrm{Mg}^{2+}$ in rat phaeochromocytoma cells. J Physiol 466:683706.

Kwan AC, Dietz SB, Webb WW, Harris-Warrick RM (2009) Activity of Hb9 interneurons during fictive locomotion in mouse spinal cord. J Neurosci 29:11601-11613.

Li WC, Soffe SR, Roberts A (2002) Spinal inhibitory neurons that modulate cutaneous sensory pathways during locomotion in a simple vertebrate. J Neurosci 22:10924-10934.

Li WC, Soffe SR, Roberts A (2004) Glutamate and acetylcholine corelease at developing synapses. Proc Natl Acad Sci U S A 101:15488-15493.

Li WC, Soffe SR, Wolf E, Roberts A (2006) Persistent responses to brief stimuli: feedback excitation among brainstem neurons. J Neurosci 26:4026-4035.

Li WC, Roberts A, Soffe SR (2009) Locomotor rhythm maintenance: electrical coupling among premotor excitatory interneurons in the brainstem and spinal cord of young Xenopus tadpoles. J Physiol 587:1677-1693.

Lieske SP, Ramirez JM (2006) Pattern-specific synaptic mechanisms in a multifunctional network. II. Intrinsic modulation by metabotropic glutamate receptors. J Neurophysiol 95:1334-1344.

MacLean JN, Schmidt BJ, Hochman S (1997) NMDA receptor activation triggers voltage oscillations, plateau potentials and bursting in neonatal rat lumbar motoneurons in vitro. Eur J Neurosci 9:2702-2711.

Marder E, Bucher D (2007) Understanding circuit dynamics using the stomatogastric nervous system of lobsters and crabs. Annu Rev Physiol 69:291-316

Marder E, Bucher D, Schulz DJ, Taylor AL (2005) Invertebrate central pattern generation moves along. Curr Biol 15:R685-R699.

Mayer ML, Westbrook GL, Guthrie PB (1984) Voltage-dependent block by $\mathrm{Mg}^{2+}$ of NMDA responses in spinal cord neurones. Nature 309:261-263.

Nieuwkoop PD, Faber J (1956) Normal tables of Xenopus laevis (Daudin). Amsterdam: North Holland.

Noga BR, Kriellaars DJ, Jordan LM (1991) The effect of selective brainstem or spinal cord lesions on treadmill locomotion evoked by stimulation of the mesencephalic or pontomedullary locomotor regions. J Neurosci 11:1691-1700.

Ozaki S, Yamada T, Iizuka M, Nishimaru H, Kudo N (1996) Development of locomotor activity induced by NMDA receptor activation in the lumbar spinal cord of the rat fetus studied in vitro. Brain Res Dev Brain Res 97:118-125.

Prime L, Pichon Y, Moore LE (1999) N-Methyl-D-aspartate-induced oscillations in whole cell clamped neurons from the isolated spinal cord of Xenopus laevis embryos. J Neurophysiol 82:1069-1073.
Ramirez JM, Tryba AK, Peña F (2004) Pacemaker neurons and neuronal networks: an integrative view. Curr Opin Neurobiol 14:665-674.

Reith CA, Sillar KT (1998) A role for slow NMDA receptor-mediated, intrinsic neuronal oscillations in the control of fast fictive swimming in Xenopus laevis larvae. Eur J Neurosci 10:1329-1340.

Rioult-Pedotti MS (1997) Intrinsic NMDA-induced oscillations in motoneurons of an adult vertebrate spinal cord are masked by inhibition. J Neurophysiol 77:717-730.

Roberts A, Li WC, Soffe SR, Wolf E (2008) Origin of excitatory drive to a spinal locomotor network. Brain Res Rev 57:22-28.

Sakurai A, Katz PS (2003) Spike timing-dependent serotonergic neuromodulation of synaptic strength intrinsic to a central pattern generator circuit. J Neurosci 23:10745-10755.

Samara RF, Currie SN (2008) Electrically evoked locomotor activity in the turtle spinal cord hemi-enlargement preparation. Neurosci Lett 441:105109.

Sautois B, Soffe SR, Li WC, Roberts A (2007) Role of type-specific neuron properties in a spinal cord motor network. J Comput Neurosci 23:59-77.

Sillar KT, Simmers AJ (1994) Electrical coupling and intrinsic neuronal oscillations in Rana temporaria spinal cord. Eur J Morphol 32:293-298.

Soffe SR (1989) Roles of glycinergic inhibition and $N$-methyl-D-aspartate receptor mediated excitation in the locomotor rhythmicity of one half of the Xenopus embryo central nervous system. Eur J Neurosci 1:561-571.

Soffe SR, Roberts A (1989) The influence of magnesium ions on the NMDA mediated responses of ventral rhythmic neurons in the spinal cord of Xenopus embryos. Eur J Neurosci 1:507-515.

Soffe SR, Roberts A, Li WC (2009) Defining the excitatory neurons that drive the locomotor rhythm in a simple vertebrate: insights into the origin of reticulospinal control. J Physiol 587:4829-4844.

Soto-Treviño C, Rabbah P, Marder E, Nadim F (2005) Computational model of electrically coupled, intrinsically distinct pacemaker neurons. J Neurophysiol 94:590-604.

Stein PS, McCullough ML, Currie SN (1998) Reconstruction of flexor/extensor alternation during fictive rostral scratching by two-site stimulation in the spinal turtle with a transverse spinal hemisection. J Neurosci 18:467-479.

Tazerart S, Vinay L, Brocard F (2008) The persistent sodium current generates pacemaker activities in the central pattern generator for locomotion and regulates the locomotor rhythm. J Neurosci 28:8577-8589.

Thörn Pérez C, Hill RH, El Manira A, Grillner S (2009) Endocannabinoids mediate tachykinin-induced effects in the lamprey locomotor network. J Neurophysiol 102:1358-1365.

Tresch MC, Kiehn O (2000) Motor coordination without action potentials in the mammalian spinal cord. Nat Neurosci 3:593-599.

Wallén P, Grillner S (1985) The effect of current passage on N-methyl-Daspartate-induced, tetrodotoxin-resistant membrane potential oscillations in lamprey neurons active during locomotion. Neurosci Lett 56:87-93.

Wallén P, Grillner S (1987) N-Methyl-D-aspartate receptor-induced, inherent oscillatory activity in neurons active during fictive locomotion in the lamprey. J Neurosci 7:2745-2755.

Wilson JM, Hartley R, Maxwell DJ, Todd AJ, Lieberam I, Kaltschmidt JA, Yoshida Y, Jessell TM, Brownstone RM (2005) Conditional rhythmicity of ventral spinal interneurons defined by expression of the Hb9 homeodomain protein. J Neurosci 25:5710-5719.

Ziskind-Conhaim L, Wu L, Wiesner EP (2008) Persistent sodium current contributes to induced voltage oscillations in locomotor-related hb9 interneurons in the mouse spinal cord. J Neurophysiol 100:2254-2264. 